\title{
Abordagens avaliativas de usabilidade em produtos editoriais digitais
}

Usability evaluation approaches in digital editorial products

Bruno Cavalheiro Bertagnolli

Universidade Estadual de Santa Catarina

cb.bruno@gmail.com su

\section{PROJËTICA}

\section{COMO CITAR ESTE ARTIGO:}

BERTAGNOLLI, Bruno Cavalheiro. Abordagens avaliativas de usabilidade em produtos editoriais digitais. Projética, Londrina, v. 11, n. 2, p. 35-59, 2020.

DOI: 10.5433/2236-2207.2020v11n2p35

Submissão: $25-07-2018$

Aceite: 05-08-2019 
RESUMO: Analisar mídias tradicionalmente impressas em meio digital passa pelo processo de avaliação de usabilidade. Assim, a partir de uma revisão bibliográfica sistemática, apresenta-se as principais questões ligadas aos processos de avaliação de usabilidade voltados para jornais, revistas, livros e plataformas de distribuição, demonstrando também o estágio atual de pesquisas relativas à usabilidade para tais produtos. Aponta-se que avaliações somativas e que tratam de bibliotecas digitais são predominantes, sendo o objetivo dos estudos a verificação do grau de usabilidade geral dos produtos. Os métodos mais utilizados são questionários e análise de tarefas, e os critérios de usabilidade baseiam-se na literatura tradicional. Conclui-se que novos estudos podem explorar diversas lacunas como heurísticas, protocolos e questionários específicos para avaliação de tais produtos, comparativos entre a usabilidade de plataformas e acessibilidade.

Palavras-chave: Usabilidade. Avaliação de interfaces. Convergência digital. Métodos.

ABSTRACT: Analyzing the media that is traditionally printed and move to the digital environment requires a process of usability evaluation. Thus, this study presents a systematic bibliographic review with the main issues related to the usability evaluation processes for newspapers, magazines, books and distribution platforms, and also demonstrates the current stage of usability research for such products. It highlights that summative evaluations and digital libraries are predominant, including studies whose purpose is to verify the degree of general usability of those products. The most used methods are questionnaires and task analysis, and usability criteria are based on traditional literature. It is concluded that new studies can explore several gaps such as specific heuristics, protocols and questionnaires to evaluate such products, comparing the usability and accessibility of the platforms.

Keywords: Usability. Interface evaluation. Digital convergence. Methods. 


\section{INTRODUÇÃO}

O desenvolvimento de novas tecnologias levou ao fenômeno de convergência das mídias impressas através de mudanças no suporte, produção e distribuição de seus produtos e serviços. Isso trouxe desafios para a indústria de comunicação originalmente surgida na era analógica, já que livros, jornais e revistas e suas formas de distribuição hoje estão presentes dentro do contexto multiplataforma. A interação com tais produtos e sistemas ocorre através da interface, e entender os fenômenos interativos entre usuário e produto é um dos focos da Interação Humano-Computador e Usabilidade.

Avaliar interfaces se justifica por fornecer aspectos relevantes para a determinação da qualidade de produtos digitais. Os métodos de usabilidade são essenciais para essa tarefa, mas a diversidade de abordagens destinadas a classes específicas de produtos traz a necessidade de estudos de levantamento mais detalhados sobre abordagens de avaliação e resultados alcançados de acordo com os processos utilizados.

Estudos que fazem a compilação de métodos de avaliação de usabilidade são diversos, mas há carência de estudos focados na revisão de métodos voltados para a avaliação de produtos editoriais, sendo essa uma das motivações deste artigo.

Assim, este trabalho procura analisar a partir de um processo sistemático de revisão de literatura quais processos são utilizados e os resultados obtidos para avaliação de usabilidade de produtos editoriais digitais como livros, revistas, jornais e suas plataformas de leitura e distribuição. Entre as questões que se pretende responder estão qual a predominância do tipo de produto e suporte avaliado, qual o objetivo dos estudos, que bases teóricas de usabilidade são utilizadas, que métodos e protocolos de avaliação são usados e como são tratados os dados 
derivados das avaliações. A análise desse escopo procura também verificar qual o patamar de estudos destinados a avaliar a qualidade de tais produtos, buscando verificar se há algum tipo de especificidade nas abordagens de avaliação e quais as contribuições trazidas para a área de ergonomia/usabilidade.

Como contribuições, o artigo pretende apontar para a possibilidade de novos estudos que explorem lacunas ainda não aprofundadas, trazer contribuições para a área de I.H.C. e apontar para o uso de métodos avaliativos mais eficientes que levem ao desenvolvimento de produtos editoriais digitais mais usáveis.

\section{MÍDIA IMPRESSA E CONVERGÊNCIA DIGITAL}

A fase analógica da comunicação iniciou com o registro artesanal dos primeiros documentos pelos escribas (HAMELINK, 1997), mas o advento da prensa gráfica em 1945 com a impressão da Bíblia de Gutemberg marcou o desenvolvimento dessa era. No século XVI, a gravação em placas de cobre permitiu a impressão de imagens realistas no papel, ampliando as possibilidades gráficas e dando origem a novos veículos impressos como as revistas (FEBVRE; MARTIN, 1999).

Todo esse processo possibilitou uma revolução do conhecimento abrindo assim a era da comunicação social. Hamelink (1997) destaca que foi a partir das inovações tecnológicas criadas na Revolução Industrial, com tecnologias baseadas na eletricidade, que a área se transformou consideravelmente.

Glover e Grant (2009) colocam que a fase analógica das tecnologias de informação e comunicação durou assim até início da década de 1980, onde inicia o processo de sobreposição das tecnologias digitais aplicada inicialmente nos processos de produção. 
Esse contexto de mudanças marcou o início da chamada cultura de convergência, termo cunhado por Henry Jenkins (2006) descreve que a convergência envolve uma transformação tanto na forma de produzir quanto na forma de consumir os meios de comunicação, tendo em suas raízes as tecnologias de informação e comunicação (TICS), mostrando a importância da computação reforçada pela popularização da internet, que possibilita a globalização do acesso à informação e comunicação.

De Las Heras (2000) aponta que a era digital criou um espaço democrático de convivência, onde as versões físicas de produtos e instituições ganharam a coexistência e a influência das suas versões digitais. Assim, começaram a surgir as versões digitais de produtos e serviços, incluindo aqueles ligados aos meios de comunicação, onde as mídias tradicionais também passam por um processo de descentralização de suporte, levando à criação de livros, jornais, revistas digitais e seus meios de distribuição como bibliotecas e bancas digitais. Embora a convergência de tais mídias tenha iniciado ainda na década de 1970, os grandes grupos tradicionais de comunicação só apostaram firmemente no texto eletrônico a partir dos primeiros anos da década de 1990, de forma que esse novo modelo de negócio tem pouco mais de duas décadas de existência efetiva (PRYOR, 2002).

Assim, o fenômeno de desmaterialização da mídia impressa a partir de sua digitalização leva à fase onde a interface dos dispositivos se torna o ponto fundamental de interação com o conteúdo (CHARTIER, 2007).

Para avaliar a qualidade de uso de jornais, livros e revistas em meio digital, é necessário observar seus sistemas de distribuição e acesso, pois na maioria das vezes a interface de navegação desses veículos é integrada dentro desses sistemas. No caso dos livros, são distribuídos e acessados via interface de bibliotecas digitais, as revistas via banca digital ou aplicativo específico e, jornais estão em formato de portal, dentro de aplicativos ou bancas digitais. 


\section{USABILIDADE E AVALIAÇÃO DE INTERFACE EM PRODUTOS DIGITAIS}

A ISO 9241 (ASSOCIAÇÃO BRASILEIRA DE NORMAS TÉCNICAS, 2012) descreve que usabilidade é "a capacidade que apresenta um sistema interativo de ser operado, de maneira eficaz, eficiente e agradável, em um determinado contexto de operação, para a realização das tarefas de seus usuários". Para Tullis e Albert (2008), medir a usabilidade deve ser vista como olhar para toda a experiência do usuário.

As avaliações de interfaces têm foco na usabilidade. Cybis (2003) conceitua que métodos de avaliação de interfaces são procedimentos para coletar informações com objetivo de averiguar a interação de usuários com sistemas e visam principalmente avaliar o quesito usabilidade. Os testes de usabilidade possuem duas abordagens, uma envolvendo testes formais realizados como verdadeiras experiências (a fim de confirmar ou refutar hipóteses específicas), e outra que emprega um ciclo iterativo de testes (destinados a expor deficiências de usabilidade ou moldar o produto em questão).

Souza et al. (1999) colocam que a escolha do método que deve ser aplicado em um caso específico depende de vários fatores, como: o que se deseja avaliar, a disponibilidade de pessoas especialistas, ambiente e equipamento para aplicação do teste, acesso aos usuários, entre outros.

Tratando-se da classificação de métodos de avaliação de usabilidade, de forma geral, pode-se dividi-los em dois grupos (CYBIS, 1997; JORDAN, 1998):

A. Quanto à etapa de vida do produto: A avaliação do tipo formativa ocorre durante a fase de projeto e desenvolvimento do produto. Seu foco na descoberta de problemas, tendo como foco guiar o design. Já, a avaliação do tipo somativa ocorre após o produto ter sido desenvolvido, permitindo também a comparação com produtos concorrentes, e tem como objetivo realizar uma avaliação global do produto. 
B. Quanto aos envolvidos na análise: A avaliação que utiliza apenas especialistas (avaliação analítica) envolve o processo de identificação de problemas potenciais de usabilidade comparando um design de interface de usuário com as diretrizes de design de usabilidade estabelecidas (HIX et al., 2004).

A avaliação que utiliza usuários (avaliação empírica) envolve “o processo de realizar estudos empíricos relativamente pequenos e rápidos para determinar quais fatores de design específicos têm maior probabilidade de afetar o desempenho da tarefa do usuário" (HIX et al., 2004, p. 3), sendo o objetivo refinar componentes ou características para uma aplicação específica.

\section{MATERIAIS E MÉTODOS}

Considerando a necessidade de conhecer o patamar atual de estudos que abordem processos de avaliação de interfaces de produtos editoriais digitais, realizou-se uma revisão sistemática da literatura usando a abordagem sugerida por Kitchenham e Charters (2007). O autor sugere um processo de revisão que envolve três etapas com várias atividades:

a) Planejamento da revisão: definir a necessidade de uma revisão sistemática; para levantar as questões de pesquisa e definir o protocolo de revisão: dados fontes, estratégia de busca e termos, critérios de seleção de estudo, qualidade dos estudos, extração de dados e síntese de dados;

b) Realização da revisão: selecionar e revisar estudos para responder às questões de pesquisa e apresentar os resultados, discussões e conclusões;

c) Relatar a revisão: escrever os resultados da revisão e formatar o documento final. 
Assim, definiu-se que a revisão de literatura sistemática deveria englobar trabalhos com foco principal no desenvolvimento ou no processo de avaliação de usabilidade de mídias digitais como livros, jornais e revistas, assim como de suas plataformas agregadoras ou de distribuição. As bases de dados Scielo, Web of Science, Science Direct e Google Acadêmico foram escolhidas para busca por oferecerem acesso facilitado e aberto. Definiram-se também os critérios de inclusão e exclusão adotados, de acordo com a tabela 1.

Tabela 1 - Tabela de critérios de inclusão e exclusão

\begin{tabular}{ll}
\hline Critérios de inclusão & Critérios de exclusão \\
\hline - Estudos dos últimos 10 anos; & - Estudos que não tratem de avaliação de \\
- Estudos em língua portuguesa e inglesa; & usabilidade de outros produtos que não sejam \\
- Apenas artigos e teses; & editoriais e seus repositórios; \\
- Apenas estudos finalizados; & - Estudos que não deixem claro o conceito \\
- Apenas estudos com acesso ao conteúdo & de usabilidade ou processos de avaliação de \\
completo; & usabilidade utilizados para obter resultados; \\
- Estudos focados na avaliação de interface & - Estudos focados em usabilidade para \\
das versões digitais de revista, jornal, livro e & públicos com condições específicas (idosos, \\
plataformas agregadoras; & surdos, cegos, etc); \\
- Estudos com foco em conceitos e métodos de & - Estudos sem acesso aberto e gratuito \\
avaliação de Usabilidade; & - Estudos focados na avaliação de usabilidade \\
& do dispositivo de leitura \\
\hline
\end{tabular}

Fonte: Autor.

Com base no planejamento adotado, passou-se à realização da revisão, que ocorreu no mês de maio/junho de 2018. Realizou-se um detalhamento para os campos de busca, de acordo com a sintaxe que permitisse que cada um dos repositórios apresentasse os melhores resultados de acordo com os filtros, trazendo um equilíbrio na relação quantitativa versus relação com o escopo de busca. 
Abordagens avaliativas de usabilidade em produtos editoriais digitais BERTAGNOLLI, B. C.

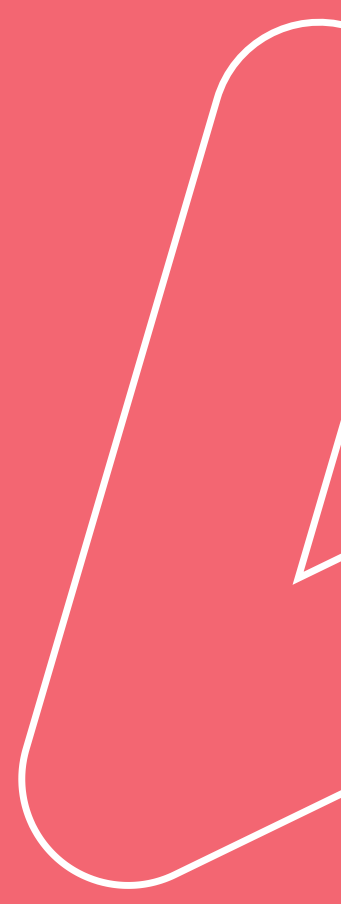

A avaliação que utiliza usuários (avaliação empírica) envolve "o processo de realizar estudos empíricos relativamente pequenos e rápidos para determinar quais fatores de design específicos têm maior probabilidade de afetar o desempenho da tarefa do usuário" (Hix et al., 2004, p.3)

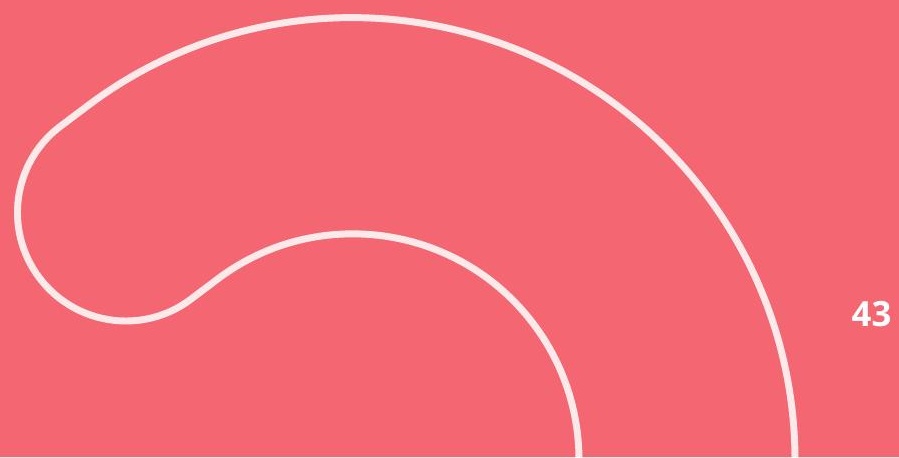


Foram definidas as strings em português e inglês integrando termos como usabilidade, livro digital, jornal digital, revista digital, biblioteca digital, banca digital, interface através de operadores de busca, sendo inicialmente encontrados como resultado 566 trabalhos que foram filtrados considerando o título. Em uma segunda filtragem, os trabalhos foram analisados mais a fundo, observando também abstract, palavras chaves e texto principal. Verificou-se que a maioria dos estudos não relacionava avaliação de usabilidade aos produtos digitais analisados e, portanto, foram descartados, chegando-se a 127 trabalhos. Uma seleção final foi realizada, considerando principalmente a pertinência dos estudos resultantes e a clareza na descrição do processo de avaliação de usabilidade empregado para avaliar as interfaces dos objetos de estudo, de forma que fosse possível tabular tais resultados. Tal etapa resultou em 28 estudos pertinentes e relevantes para análise, apresentados na tabela 2 .

Tabela 2 - Estudos escolhidos após a seleção

\begin{tabular}{|c|c|c|c|}
\hline & Autor do estudo & Título & Ano \\
\hline (1) & $\begin{array}{l}\text { JOU; TENNYSON; WANG \& } \\
\text { HUANG }\end{array}$ & $\begin{array}{l}\text { A study on the usability of E-books and APP in } \\
\text { engineering courses: A case study on mechanical } \\
\text { drawing }\end{array}$ & 2016 \\
\hline (2) & GUEVAL; TARNOW \& KUMM & $\begin{array}{l}\text { Implementing e-books: Faculty and student } \\
\text { experiences }\end{array}$ & 2015 \\
\hline (3) & ROSA \& VERAS & $\begin{array}{l}\text { Avaliação heurística de usabilidade em jornais } \\
\text { online: estudo de caso em dois sites }\end{array}$ & 2013 \\
\hline (4) & $\begin{array}{l}\text { FENSTERSEIFER, KRENING, } \\
\text { TEIXEIRA, SILVA \& SILVA }\end{array}$ & $\begin{array}{l}\text { Histórias em quadrinhos no contexto dos } \\
\text { dispositivos móveis: aspectos teóricos e análise de } \\
\text { usabilidade. }\end{array}$ & 2016 \\
\hline (5) & $\begin{array}{l}\text { ÇETIN; ÖZKARACA; SAKAL; } \\
\text { GÜVEN \& KOÇMAN }\end{array}$ & $\begin{array}{l}\text { A usability assessment of an epub } 3.0 \text { based ebook } \\
\text { developed for algorithms and programming course }\end{array}$ & 2017 \\
\hline (6) & CAETANO; SILVA \& CORREA & $\begin{array}{l}\text { Avaliação de usabilidade da interface da biblioteca } \\
\text { digital das faculdades integradas de são pedro: um } \\
\text { estudo de caso }\end{array}$ & 2012 \\
\hline (7) & HENN; FRANÇA \& DIAS & $\begin{array}{l}\text { Navegabilidade em portais: estudo com usuários } \\
\text { dos portais dos jornais o norte e jornal da Paraíba }\end{array}$ & 2010 \\
\hline
\end{tabular}




\begin{tabular}{|c|c|c|c|}
\hline (8) & ABDULLAH \& WEI & $\begin{array}{l}\text { Usability Measurement of Malaysia Online News } \\
\text { Websites }\end{array}$ & 2008 \\
\hline (9) & LIMA; OLIVEIRA \& SANTANA & $\begin{array}{l}\text { Metodologia para avaliação do nível de usabilidade } \\
\text { de bibliotecas digitais: um estudo na Biblioteca } \\
\text { Virtual de Saúde }\end{array}$ & 2013 \\
\hline (10) & BOHMERWALD & $\begin{array}{l}\text { Uma proposta metodológica para avaliação de } \\
\text { bibliotecas digitais: usabilidade e comportamento } \\
\text { de busca por informação na Biblioteca Digital da } \\
\text { PUC-Minas }\end{array}$ & 2005 \\
\hline (11) & PEREIRA & $\begin{array}{l}\text { Avaliação de usabilidade em bibliotecas digitais: } \\
\text { um estudo de caso }\end{array}$ & 2011 \\
\hline (12) & LACERDA & $\begin{array}{l}\text { Análise de usabilidade de Livro Digital na Ead: Uma } \\
\text { Avaliação Heurística }\end{array}$ & 2014 \\
\hline (13) & GOLFETTO; BALDESSAR & $\begin{array}{l}\text { Análise de uso e propostas para aprimoramento } \\
\text { de edições digitais de revistas através de grupos } \\
\text { focais }\end{array}$ & 2014 \\
\hline (14) & MASREK \& KHAN & $\begin{array}{l}\text { Usability Assessment of Web Digital Library: A } \\
\text { Survey Among Undergraduate Students }\end{array}$ & 2015 \\
\hline (15) & $\begin{array}{l}\text { ANN BLANDFORD \& GEORGE } \\
\text { BUCHANAN }\end{array}$ & $\begin{array}{l}\text { Usability of digital libraries: a source of creative } \\
\text { tensions with technical developments }\end{array}$ & 2003 \\
\hline (16) & ALASEM & $\begin{array}{l}\text { Evaluating the Usability of Saudi Digital Library's } \\
\text { Interface (SDL) }\end{array}$ & 2013 \\
\hline (17) & $\begin{array}{l}\text { SAID; CHRISTINE; ANAS; } \\
\text { COLIN; ALBAN; ANDREW; } \\
\text { CAMILLE; DOTAN, ACADIA }\end{array}$ & AUP library web site usability study & 2010 \\
\hline (18) & PEKKALA \& KUHNA & Usability of digital magazines & 2012 \\
\hline (19) & CHANLIN \& HUNG & Usability and evaluation of a library mobile web site & 2016 \\
\hline (20) & KHAN; ZAHID \& RAFIQ & $\begin{array}{l}\text { Usability Evaluation of University of Management } \\
\text { and Technology Library, Lahore Website: A survey } \\
\text { of user satisfaction }\end{array}$ & 2014 \\
\hline (21) & HÄMÄLÄINEN & $\begin{array}{l}\text { User feedback and development of an online } \\
\text { magazine platform }\end{array}$ & 2017 \\
\hline$(22)$ & $\mathrm{JOO}(1)$ & $\begin{array}{l}\text { How are usability elements - eficiency, } \\
\text { efectiveness, and satisfaction - correlated with } \\
\text { each other in the context of digital libraries? }\end{array}$ & 2011 \\
\hline (23) & $\mathrm{JOO}(2)$ & $\begin{array}{l}\text { Measuring the usability of academic digital } \\
\text { libraries: Instrument development and validation }\end{array}$ & 2017 \\
\hline (24) & IQBAL \& WARRAICH & $\begin{array}{l}\text { Usability evaluation of an academic library website: } \\
\text { A case of the University of the Punjab }\end{array}$ & 2012 \\
\hline
\end{tabular}




\begin{tabular}{llll}
\hline (25) & PATERSON \& HIGHLIGHT & Usability Inspection of Digital Libraries & 2018 \\
\hline (26) & RADAIDEH \& SHANAB & $\begin{array}{l}\text { Usability Evaluation of Online News Websites: A } \\
\text { User Perspective Approach }\end{array}$ & 2011 \\
\hline (27) & JOO; LIN \& LU & A Usability Evaluation Model for Academic Library & 2011 \\
& & Websites: Efficiency, Effectiveness and Learnability & \\
\hline (28) & WEl; LIN2 \& YEH & Effects of touch screen mobile devices and e-book & 2017 \\
& & systems on students reading performance: A & \\
& & usability evidence & \\
\hline
\end{tabular}

Fonte: Autor. cinco questões formuladas de forma a responder ao problema de pesquisa:

Tabela 3 - Questões de Pesquisa

\begin{tabular}{l}
\hline Questões de pesquisa \\
\hline 1. O estudo avalia qual produto e suporte? \\
- Qual é o objeto de estudo? \\
- Para qual suporte digital o estudo é dirigido? \\
\hline 2. Qual o objetivo do estudo? \\
- O estudo conclui para qual apontamento? \\
\hline 3. Qual base teórica de usabilidade adotada no estudo? \\
- Em quais autores é baseada a conceituação de usabilidade adotada? \\
- Quais são os critérios/métricas adotadas? \\
\hline 4. Quais são os tipos e métodos de avaliação adotados? \\
- Que tipo de avaliação é adotada? \\
• Quais métodos e protocolos utilizados para as avaliações? \\
• Em que fase de vida do produto a avaliação é realizada? Qual o número de envolvidos nos testes \\
\hline 5. Que tipo de tratamento utilizado para análise dos dados colhidos? \\
• Como o estudo trabalha com os dados colhidos?
\end{tabular}

Fonte: Autor. 


\section{RESULTADOS}

As informações extraídas sobre os estudos foram agrupadas, resumidas e tabuladas, apresentando-se a seguir a análise.

\section{Questão 1: Produtos e suportes avaliados}

Em relação ao tipo de produto analisado, é possível verificar em qual patamar os estudos atuais encontram-se para cada tipo de produto editorial, apontando para possíveis lacunas que podem ser exploradas em relação ao objeto de análise. A avaliação dos suportes estudados permite verificar como os estudos de usabilidade levam em consideração a realidade multiplataforma de suportes de leitura, se tais especificidades são consideradas e levam a algum tipo de processo avaliativo.

- Qual é o objeto de estudo?

A maioria dos estudos são dirigidos a bibliotecas digitais (15 estudos, $44 \%$ ), seguidos por aqueles que avaliam a interface de livros digitais (6 estudos, 17\%), jornais digitais (5 estudos, 14\%) e revistas (3 estudos, 9\%). Apenas um estudo era voltado a bancas digitais. A maior exploração de bibliotecas digitais pode ser relacionada ao tempo de mercado e pioneirismo como serviço agregador de conteúdo editorial disponibilizados na web, levando também aos estudos de livros digitais. Em relação às revistas e sistemas agregadores como as bancas digitais - percebe-se carência de estudos, que pressupõe a possibilidade de análises relacionadas ao seu uso e interface.

- Para qual suporte digital o estudo é dirigido? (smartphone, computador, tablet, etc.)

A maioria dos trabalhos não menciona para qual plataforma é dirigido, inferindo-se que sejam voltados para computadores (vinte e dois estudos, 88\%). Alguns direcionam-se para tecnologias mobile (três estudos, 10\%), não havendo uma especificação ou tratamento metodológico voltado a explorar diferenças 
entre plataformas. Dos que exploram especificidades, três abordam tablets e um aborda smartphone. Isso leva a crer que há abertura para explorar entre diferentes dispositivos e suas relações com fatores de I.H.C. e usabilidade.

\section{Questão 2: Objetivos dos estudos}

Tal questão aponta em que direção ou para que propósito as avaliações são realizadas. Os objetivos gerais foram classificados em cinco categorias, sendo que alguns estudos apresentam mais de um objetivo. Verificou-se que a maioria das ocorrências relaciona-se à abordagem clássica de aferir o nível de usabilidade do produto analisado (12 estudos, 39\%). Outro objetivo recorrente foi verificar problemas de usabilidade, sendo algumas vezes acompanhado pela sugestão de soluções para tais problemas (4 estudos, 16\%). Os trabalhos destinados à criação de diretrizes para avaliação de usabilidade têm o propósito de criar requisitos de avaliação geral para os produtos analisados (2 estudos, 6\%). A validação de processos de avaliação ou criação de questionários que medem a usabilidade do produto é o objetivo de quatro estudos (14\%). Não classificados dentro das categorias anteriores devido à especificidade de seus objetivos e com frequência única, encontram-se estudos com o objetivo de verificar a satisfação do usuário; comparar o nível de usabilidade entre produtos; verificar correlação entre critérios de usabilidade e identificar especificidades entre interação e navegação.

\section{Questão 3: Base teórica de usabilidade adotada}

A análise da base teórica utilizada procura demonstrar sobre quais autores é baseada a conceituação e critérios de usabilidade utilizados nos estudos, considerando as diversas vertentes da literatura sobre o tema.

- Em quais autores é baseada a conceituação de usabilidade adotada?

A escolha entre os autores ajuda a entender sob qual prisma a usabilidade é utilizada e também leva à definição da escolha dos modelos de métricas ou variáveis de medição adotadas para a avaliação de usabilidade realizada. Alguns 
trabalhos baseiam-se em uma única base teórica/autor, enquanto outros utilizam duas ou mais para fundamentar o estudo. Nielsen e a norma ISO (18 estudos, 50\%) são as bases predominantes. Há trabalhos que não mencionam a base teórica de usabilidade (5 estudos, 14\%), muitas vezes sendo baseados nos conhecimentos dos próprios autores ou em experimentos feitos com usuários com o objetivo de levantar questões que são convertidas em diretrizes ou modelos de avaliação. Treze estudos (36\%) baseiam-se em algum dos seguintes autores: Davis (1989); Bertini et al.; Nascimento e Amaral (2010); Fleming (1998); Morville e Rosenfeld (2006); Krug (2005); Shneiderman (1987); Choo, Detlor e Turnbull (1999); Prechansky (2011); Harrison et al. (2013); Seffah et al. (2001, 2006); Bevan (2000); Jeng (2005); Tsakonas e Papatheodorou (2006); Britton (2015); Oulanov e Pajarillo (2001).

- Quais são os critérios/métricas de usabilidade adotadas?

Considerando os diversos modelos de critérios, métricas e princípios de usabilidade existentes na literatura, o objetivo foi verificar a frequência que são utilizados. Através da análise, foi possível perceber que muitos critérios, embora tenham propósito de avaliar questões semelhantes, recebem nomes diferentes.

Tabela 4 - Critérios de usabilidade adotados e frequência de estudos que os utilizam

\begin{tabular}{ll}
\hline Critérios & Frequência \\
\hline 8 Heurísticas de Bertini; 7 princípios de Prechansky; Compatibilidade; Intenção de & 1 \\
uso; Gestão do tempo; Responsividade; Acessibilidade; Busca; Ajuda; Terminologia & \\
\hline Recursos; Controle; Afeto & 2 \\
\hline Funcionalidade; Facilidade de uso; Legibilidade & 3 \\
\hline Utilidade & 4 \\
\hline 10 Heurísticas de Nielsen; Navegação & 5 \\
\hline Organização & 6 \\
\hline Aprendizagem & 8 \\
\hline Interface; Satisfação & 9 \\
\hline Eficácia & 10 \\
\hline Eficiência & 14 \\
\hline
\end{tabular}

Fonte: Autor. 
O uso de critérios de usabilidade é influenciado pelo método de avaliação utilizado, como nos estudos que realizam avaliação heurística baseada em algum modelo, ou questionários já consolidados na literatura que propõem seus critérios. Trabalhos que se baseiam em abordagens de avaliação de usabilidade totalmente qualitativas como entrevistas ou Focus Group, também não utilizam critérios, mostrando outro tipo de abordagem.

\section{Questão 4: Tipo e Métodos de avaliação adotados}

O tipo de avaliação adotada, com especialistas, usuários ou ambos, demonstra sobre qual perspectiva e aprofundamento os produtos são avaliados. Os métodos são o meio pelo qual se obtém os dados para formular as respostas aos problemas de pesquisa propostos nos estudos, sendo que a análise de como são utilizados aponta para os melhores procedimentos avaliativos se há especificidades considerando os produtos editoriais.

- Que tipo de avaliação é adotada?

Dos estudos, 22 (79\%) utilizam usuários (empírica), 3 (11\%) utilizam apenas especialistas e $3(11 \%)$ utilizam tanto usuários como especialistas.

- Quais métodos utilizados para as avaliações?

Considerando a diversidade de métodos para avaliação de usabilidade, a frequência de uso ajuda a entender quais são as abordagens mais utilizadas e populares. Em frequência decrescente de uso aparecem os Questionários (18 estudos, 45\%), Análise de Tarefas (10 estudos, 25\%), Avaliação Heurística (6 estudos, 15\%), Focus Group (3 estudos, 8\%), Entrevista (2 estudos, 6\%) e apenas um estudo utilizou Inspeção.

- Em que fase de vida do produto a avaliação é realizada?

Essa questão ajuda a entender se os testes de usabilidade são utilizados para criar produtos melhores ou melhorar os já existentes. A maioria dos estudos 
realiza avaliação de produtos finalizados (avaliação somativa, 25 estudos, 86\%), poucos se dedicam a avaliar produtos em fase de desenvolvimento (avaliação formativa, 4 estudos, 14\%).

- Qual o número de envolvidos nos testes?

A análise da amostragem de participantes, sejam especialistas ou usuários, depende do uso e combinação dos métodos utilizados, e mostra o número de envolvidos usualmente utilizados.

Tabela 5 - Número e tipo de avaliador envolvido por método utilizado

\begin{tabular}{llllll}
\hline Questionários & $\begin{array}{l}\text { Avaliação } \\
\text { Especialista }\end{array}$ & $\begin{array}{l}\text { Focus } \\
\text { Group }\end{array}$ & Tarefas & Entrevista & Inspeção \\
\hline $\begin{array}{l}\text { 8-22 usuários } \\
\text { (4 estudos) }\end{array}$ & (3 especialista & $\begin{array}{l}6 \text { usuários } \\
\text { (1 estudo) }\end{array}$ & $\begin{array}{l}\text { 8-15 usuários } \\
\text { (5 estudos) }\end{array}$ & $\begin{array}{l}8 \text { usuários } \\
\text { (1 estudo) }\end{array}$ & $\begin{array}{c}\text { 1 especialista } \\
\text { (1 estudo) }\end{array}$ \\
\hline 30-60 usuários & 2 especialistas & $\begin{array}{l}12 \text { usuários } \\
\text { (5 estudos) }\end{array}$ & $\begin{array}{l}\text { (1 estudo) } 50 \text { usuários } \\
\text { (3 estudos) }\end{array}$ & $\begin{array}{l}\text { 4 usuários } \\
\text { (1 estudo) }\end{array}$ & \\
\hline 80-222 & 3 especialistas & 9 usuários & 53-60 usuários & & \\
(5 estudos) & (2 estudos) & (1 estudo) & (2 estudos) & & \\
\hline 230-336 & & & & & \\
(4 estudos) & & & & & \\
\hline
\end{tabular}

Fonte: Autor.

Estudos que utilizam avaliação heurística representam as menores amostras, já os estudos que utilizam questionários online são aqueles que envolvem o maior número de pessoas nos testes.

\section{Questão 5: Tratamento utilizado para análise dos dados colhidos}

A resposta a essa questão mostra de que forma os estudos trabalham para aferir os resultados respondendo ao problema de pesquisa proposto. Foram categorizados dois tratamentos: análise qualitativa (sem emprego de métodos estatísticos) e análise quali/quantitativa (com emprego de métodos estatísticos). 
- Como o estudo trabalha com os dados colhidos?

Necessariamente trabalhar com avaliação de usabilidade passa por uma análise qualitativa dos dados. Do total de estudos, 16 (55\%) trataram apenas de forma qualitativa os dados colhidos a partir de processos de avaliação de usabilidade. A outra parte, 13 estudos (45\%), conjugaram uma análise qualitativa (estatística) e após uma análise qualitativa.

\section{CONCLUSÃO E TRABALHOS FUTUROS}

A presente pesquisa por meio de revisão sistemática apresentou um levantamento apontando o patamar atual e questões abordadas em estudos que exploram avaliação de usabilidade de produtos editoriais digitais.

Conclui-se que bibliotecas e livros digitais são o foco da maioria dos estudos, possivelmente por estarem ligados a sistemas universitários e estarem mais próximos da realidade dos próprios pesquisadores. Há carência de estudos que explorem revistas e bancas digitais sob o prisma da usabilidade. Quanto ao objetivo dos estudos, $40 \%$ procuram verificar o grau de usabilidade dos produtos ou descobrir problemas (alguns sem propor soluções - 13\%). Apenas 19\% procuram criar ou validar ferramentas para avaliação dos produtos relatados ou propor o uso da avaliação de usabilidade para propósitos diferenciados. Nielsen e as normas da Isonorm são base teórica de usabilidade de mais da metade dos estudos, mostrando a consolidação e confiança de tais literaturas. A compilação de autores também é utilizada de forma expressiva, de forma a buscar o modelo mais adequado dependendo das questões e contexto de pesquisa. Consequentemente, os critérios de usabilidade mais frequentes derivam da literatura de Nielsen e Isonorm, como eficiência (22\%), eficácia (16\%), satisfação (14\%). Também se verificou critérios trazidos por outras literaturas ou criados pelos próprios pesquisadores. Os usuários são avaliadores em $70 \%$ dos estudos, por meio de 
realização de tarefas e questionários em produtos já finalizados. Apenas $14 \%$ dos estudos realizam avaliações formativas (produtos ainda em desenvolvimento). 0 número de avaliadores é relacionado ao tipo de método adotado: questionários ou tarefas online possibilitam grandes amostragens, já em abordagens presenciais o número é restrito. Mais da metade dos estudos (55\%) não usam tratamentos estatísticos para validar os resultados, aplicando um caráter qualitativo em suas conclusões.

Conclui-se que tanto pelo quantitativo como pelas abordagens, o patamar de estudos destinados a avaliar a qualidade de produto digitais relacionados a mídias editoriais ainda é bastante incipiente, abrindo-se possibilidades de abordagem de diversos problemas que podem ter contribuições significantes para a área de ergonomia/usabilidade. Entre as possibilidades estão estudos que apontem para a criação de heurísticas específicas para tais produtos, a criação de protocolos de avaliação, questionários específicos, comparativos entre a usabilidade de plataforma impressa e digital considerando as especificidades de público-produto, acessibilidade, entre outros. 
Projética, Londrina, v.11, n.2, p. 35-59, agosto 2020

\section{REFERÊNCIAS}

1. ABDULLAH, Rusli; WEI, Koh Tieng. Usability measurement of malaysia online news websites. International Journal of Computer Science and Network Security, Seoul, v. 8, n. 5, p. 159-166, 2008.

2. ALASEM, Abdulrahman. Evaluating the Usability of Saudi Digital Library's Interface. Proceedings of the World Congress on Engineering and Computer, San Francisco, v. 1, p. 23-25, 2013.

3. ASSOCIAÇÃO BRASILEIRA DE NORMAS TÉCNICAS. NBR ISO 9241: ergonomia da interação humano-sistema. Parte 100: introdução às normas relacionadas à ergonomia de software. Rio de Janeiro: ABNT, 2012.

4. BLANDFORD, Ann; BUCHANAN, George. Usability of digital libraries: a source of creative tensions with technical developments. IEEE Technical Committee on Digital Libraries Bulletin, Birmingham, AL, v. 1, n. 1, p. 1-9, 2003.

5. BLANDFORD, Ann; BUCHANAN, George. Usability of digital libraries: a source of creative tensions with technical developments.

6. BOHMERWALD, Paula. Uma proposta metodológica para avaliação de bibliotecas digitais: usabilidade e comportamento de busca por informação na Biblioteca Digital da PUC-Minas. Ciência da Informação, Brasília, v. 34, n. 1, p. 95-103, 2005.

7. CAETANO, Alessandra Monteiro Pattuzzo; SILVA, Daniela Lucas; CORREA, Suelisbete Pinto. Avaliação de usabilidade da interface da Biblioteca Digital das Faculdades Integradas São Pedro: um estudo de caso. Revista Científica Faesa, Vitória, v. 8, n. 1, p. 65-85, 2012. 
8. ÇETIN, Güercan; Özkaraca, Osman; SAKAL, Murat; GÜVENÇ, Ercüment. A usability assessment of an epub 3.0 based ebook developed for algorithms and programming course. Information Technologies and Learning Tools, Kyiv, v. 58, n. 2, p. 129-140, 2017. Disponível em: https://journal.iitta.gov.ua/ index.php/itlt/article/view/1576. Acesso em: 20 jan. 2018.

9. CHANLIN, Lih-Juan; HUNG, Wei-Hsiang. Usability and evaluation of a library mobile web site. The Electronic Library, Oxford, v. 34, n. 4, p. 636-650, 2016.

10. CHARTIER, Roger. Os livros resistirão às tecnologias digitais. [Depoimento a Cristina Zahar]. Revista Nova Escola, São Paulo, n. 204, ago. 2007. Disponível em: http://revistaescola.abril.com.br/ lingua-portuguesa/fundamentos/ roger-chartier-livros-resistirão-tecnologias-digitais-610077.shtml?page=all. Acesso em: 20 jan. 2018.

11. CYBIS, Walter de Abreu. Abordagem ergonômica para IHC. Florianópolis, SC: Laboratório de Utilizabilidade da Informática, Universidade Federal de Santa Catarina, 1997.

12. CYBIS, Walter de Abreu. Engenharia de usabilidade: uma abordagem ergonômica. Florianópolis, SC: Laboratório de Utilizabilidade da Informática, Universidade Federal de Santa Catarina, 2003.

13. DE LAS HERAS, Antonio Rodríguez. Las propriedades del espacio digital. In: CONGRESO IBEROLATINOAMERICANO DE INFORMÁTICA EDUCATIVA ESPECIAL, Córdoba. Anais [...]. Córdoba: CIIEE, 2000. CD-ROM.

14. FEBVRE, Lucien; MARTIN, Henri-Jean. The coming of the book. London: Verso, 1999.

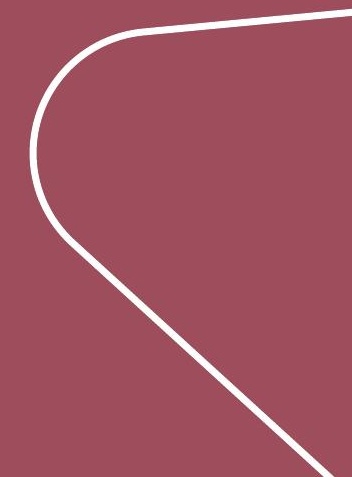


Projética, Londrina, v.11, n.2, p. 35-59, agosto 2020

15. FENSTERSEIFER, Thais Arnold; KRENING, Thiago da Silva; TEIXEIRA, Fábio Gonçalves; SILVA, Tânia Koltermann; SILVA, Régio Pierre. Histórias em quadrinhos no contexto dos dispositivos móveis: aspectos teóricos e análise de usabilidade. InfoDesign: revista brasileira de design da informação, São Paulo, v. 13, n. 1, p. 22-38, 2016.

16. GLOVER, Ian; GRANT, Peter. Digital communications. 3. ed. United Kingdom: Harlow, 2009.

17. GUEVAL, Jennifer; TARNOW, Karen; KUMM, Sharon. Implementing e-books: Faculty and student experiences. Teaching and Learning in Nursing, Amsterdam, v. 10, n. 4, p. 181-185, 2015.

18. HAMELINK, Cees J. New information and communication technologies, social development and cultural change. Geneva: United Nations Research Institute for Social Development, 1997. (Discussion Paper, n. 86.)

19. HENN, Gustavo; FRANÇA, Henrique; DIAS, Guilherme Ataíde. Navegabilidade em portais: estudo com usuários dos portais dos jornais O Norte e Jornal da Paraíba. Revista Digital de Biblioteconomia e Ciência da Informação, Campinas, v. 8, n. 1, p. 37-52, jul. /dez. 2010.

20. HIX, Deborah; GABBARD, Joseph L.; SWAN, Edward; LIVINGSTON, Mark A.; HOLLERER, Tobbias H.; JULIER, Simon J.; BAILLOT, Yohan; BROWN, Dennis. A cost-effective usability evaluation progression for novel interactive systems. In: ANNUAL HAWAII INTERNATIONAL CONFERENCE ON SYSTEM SCIENCES, 37th, 2004, Big Island, HI, USA. Proceedings [...]. New York, NY: IEEE, 2004. p. $1-10$.

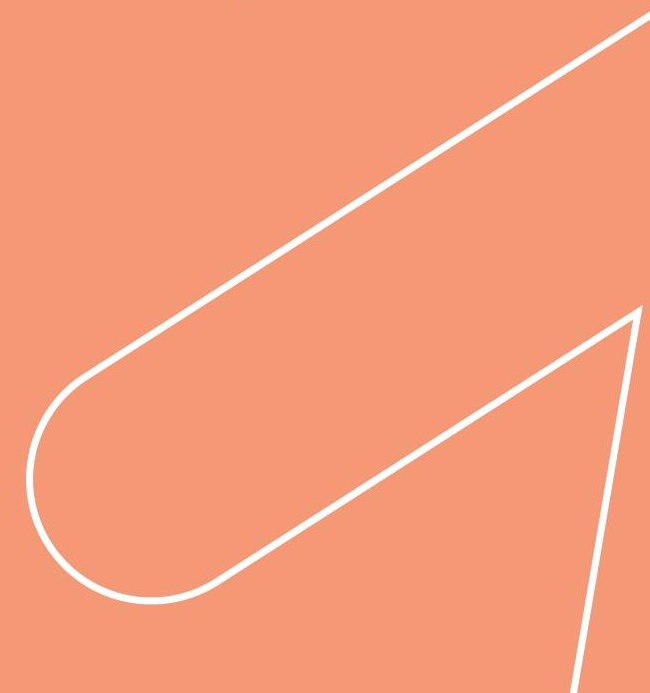


Abordagens avaliativas de usabilidade em produtos editoriais digitais BERTAGNOLLI, B. C.

21. IQBAL, Memoona; WARRAICH, Nosheen Fatima. Usability evaluation of an academic library website: a case of the university of the Punjab. Pakistan Journal of Library and Information Science, Lahore, v. 13, n. 13, 2012.

22. JENKINS, Henry. Cultura da convergência. São Paulo: Aleph, 2006.

23. JOO, Soohyung; LEE, Jee Yeon. Measuring the usability of academic digital libraries: instrument development and validation. Electronic Library, Oxford, v. 29, n. 4, p. 523-537, 2011.

24. JOO, Soohyung; LIN, Suyu; LU, Kun. A usability evaluation model for academic library websites: efficiency, effectiveness and learnability. Journal of Library and Information Studies, Taiwan, v. 9, n. 2, p. 11-26, 2011.

25. JORDAN, Patrick W. An introduction to usability. London: Taylor \& Francis, 1998.

26. JOU, Min; TENNYSON, Robert D.; WANG, Jingying; HUANG, Szu Yiang. A study on the usability of E-books and APP in engineering courses: a case study on mechanical drawing. Computers and Education, New York, v. 92, p. 181-193, 2016.

27. KHAN, Muhammad Tufail; ZAHID, Anella; RAFIQ, Muhammad. Usability evaluation of university of management and technology library, lahore website: a survey of user satisfaction. Pakistan Library \& Information Science Journal, Lahore, Pakistan, v. 45, n. 4, 2014.

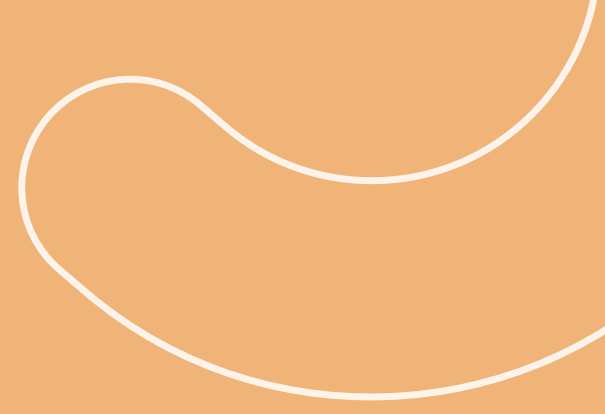


Projética, Londrina, v.11, n.2, p. 35-59, agosto 2020

28. KITCHENHAM, Barbara; CHARTERS, Stuart. Guidelines for performing systematic literature reviews in software engineering. Version 2.3. Keele, Staffs: Software Engineering Group, School of Computer Science and Mathematics, Keele University; Durham, UK: Department of Computer Science, University of Durham, 2007. EBSE Technical Report, EBSE-2007-01, p. 1-65.

29. LACERDA, Luciana Melo. Análise de usabilidade de livro digital na EaD: aplicação da avaliação heurística proposta por Pechansky. 2014. 70 f. Dissertação (Mestrado Profissional em Design) - Centro de Ciências Humanas, Letras e Artes, Universidade Federal do Rio Grande do Norte, Natal, RN, 2014.

30. LIMA, Izabel França; OLIVEIRA, Henry Poncio Cruz; SANTANA, Sérgio Rodrigues. Metodologia para avaliação do nível de usabilidade de bibliotecas digitais: um estudo na Biblioteca Virtual de Saúde. TransInformação, Campinas, v. 25, n. 2, p. 135-143, 2013.

31. MASREK, Mohamad Noorman; KHAN, Asad. Usability assessment of web digital library: a survey among undergraduate students. Journal of Information and Knowledge Management, Denton, Texas, v. 5, n. 2, p. 46-56, 2015.

32. PEREIRA, Fernanda. Avaliação de usabilidade em bibliotecas digitais: um estudo de caso. 2011. 122 f. Dissertação (Mestrado em Ciência da Informação) - Universidade Federal de Minas Gerais, Belo Horizonte, MG, 2011.

33. PRYOR, Larry. The future of news: the third wave on online journalism. Online Journalism Review, Los Angeles, CA, 18 Apr. 2002. Disponível em: http://www.ojr.org/ojr/future/1019174689.php. Acesso em: 14 mar. 2018. 
34. ROSA, Juan Miguel; VERAS, Manoel. Avaliação heurística de usabilidade em jornais online: estudo de caso em dois sites. Perspectivas em Ciência da Informação, Belo Horizonte, MG, v. 18, n. 1, p. 138-157, 2013.

35. SAID, A.; ALBAN, D.; ANAS B.; COLIN C.; ALBAN D.; ANDREW M.; CAMILLE R.; DOTAN S.; ACADIA, W. AUP Library web site usability study. CM/CS, Fall, 2010. Disponível em: https://ac.aup.edu/ croda/SampleStudentsWork/ cs348/finalProjectCS348F10/AUPLibraryWebSiteUsabilityCS248F10_Small. pdf. Acesso em: 14 mar. 2018.

36. SOUZA, Clarisse S.; PRATES, Raquel O.; BARBOSA, Simone D. J. A method for evaluating software communicability. Rio de Janeiro, RJ: Departamento de Informática, PUC-Rio, 1999. Monografias em Ciência da Computação. MCC11/99.

37. TULLIS, Thomas, ALBERT, William. Measuring the user experience: collecting, analyzing and presenting usability. San Francisco, CA: Morgan Kaufmann, 2008.

38. WEI, Chun-Chun; LIN, Yang-Cheng; YEH, Chung-Hsing. Effects of touch screen mobile devices and e- book systems on students' reading performance: a usability evidence. Journal of Engineering Technology, Johnson City, v. 6, n. 2007, p. 58-78, 2017. 